\title{
Uncertainty in velocity measurement based on diode-laser absorption in nonuniform flows
}

\author{
Fei Li, ${ }^{1}$ Xilong Yu, ${ }^{1}$ Weiwei Cai, ${ }^{2}$ and Lin $\mathrm{Ma}^{2, *}$ \\ ${ }^{1}$ State Key Lab of High Temperature Gas Dynamics, Institute of Mechanics, Chinese Academy of Sciences, \\ Beijing 100190, China \\ ${ }^{2}$ Department of Aerospace and Ocean Engineering, Virginia Tech, Blacksburg, Virginia 24061, USA \\ *Corresponding author: linma@vt.edu
}

Received 16 February 2012; revised 14 May 2012; accepted 14 May 2012; posted 14 May 2012 (Doc. ID 163180); published 9 July 2012

\begin{abstract}
This work investigates the error caused by nonuniformities along the line-of-sight in velocity measurement using tunable diode-laser absorption spectroscopy (TDLAS). Past work has demonstrated TDLAS as an attractive diagnostic technique for measuring velocity, which is inferred from the Doppler shift of two absorption features using two crossing laser beams. However, because TDLAS is line-of-sight in nature, the obtained velocity is a spatially averaged value along the probing laser beams. As a result, nonuniformities in the flow can cause uncertainty in the velocity measurement. Therefore, it is the goal of this work to quantify the uncertainty caused by various nonuniformities typically encountered in practice, including boundary layer effects, the divergence/convergence of the flow, and the methods used to fit the Doppler shift. Systematic analyses are performed to quantify the uncertainty under various conditions, and case studies are reported to illustrate the usefulness of such analysis in interpreting experimental data obtained from a scramjet facility. We expect this work to be valuable for the design and optimization of TDLAS-based velocimetry, and also for the quantitative interpretation of the measurements. (C) 2012 Optical Society of America
\end{abstract}

OCIS codes: $\quad 120.7250,280.3420,300.1030,300.6260$.

\section{Introduction}

Among all the laser diagnostics developed for combustion flows, tunable diode-laser absorption spectroscopy (TDLAS) has been demonstrated as an attractive technique offering unique advantages such as fast temporal resolution, quantitative measurements, and low cost. As a result, variations of TDLAS have been developed to monitor multiple flow parameters, including temperature, pressure, velocity, density, and flow rate, and applications of TDLAS have been demonstrated in a wide spectrum of combustion systems ranging from aircraft engine, IC engine, high enthalpy wind tunnel, and supersonic combustion flow. Readers interested in a comprehensive and in-depth discussion of the capabilities

$1559-128 \mathrm{X} / 12 / 204788-10 \$ 15.00 / 0$

(C) 2012 Optical Society of America and applications of TDLAS are referred to a review paper [1].

Despite these unique advantages, the limitation of TDLAS is well recognized: it is a line-of-sight technique in nature and hence its application is limited to flows with negligible nonuniformity. A considerable amount of research efforts have been invested in overcoming this limitation. Past efforts can be broadly divided into two categories. Efforts in the first category analyze the uncertainty caused by nonuniformities, so that measurement uncertainty can be quantified and minimized. For example, after analyzing effects of the thermal and concentration boundary layer in concentration and temperature measurements, the uncertainties caused by such a boundary layer can be quantified, and furthermore optimal wavelengths can be chosen to minimize the uncertainties [2]. The work presented here falls in this category: we analyzed the effects caused by 
nonuniformities to quantify uncertainties in velocity measurements, and these analyses also suggested ways to minimize the uncertainties. Efforts in the second category attempted to obtain spatial resolution by combining TDLAS with tomography inversion [3-5]. This approach typically requires measurements using multiple probing beams and is not the focus of this study.

In this work, we focused on analyzing the uncertainties of velocity measurements using TDLAS, especially when applied in high speed flows such as those encountered in scramjet facilities [6,7]. Velocity is a critical parameter for propulsion study, and various mechanisms can cause nonuniformity in supersonic flows. Thus, the effects of nonuniformities on velocity measurement merit careful examination. Recent work includes that performed by Chang et al. $[8,9]$, where the performance of TDLAS-based velocimetry was evaluated in a flow tunnel, and that performed by Brown and Barhorst [10], where the average flow rate in a flight test was estimated assuming nonuniform pressure distribution obtained from computational fluid dynamics (CFD) simulation. These works analyzed the velocity-error caused by boundary layers in nonreacting flow fields, and the influence of nonuniform pressure because of the pressure-induced frequency-shift. These past results were usually application-specific, and therefore this work aims at providing a more general analysis based these past efforts. The approach adopted in this work is to analyze the effects of nonuniformities in general quasi-two-dimensional flows, and the methodology is to decouple the nonuniformities into those normal to the overall flow direction (e.g., those caused by boundary layer effects), and those along the overall flow direction (e.g., those caused by flow divergence/convergence or heat transfer). The results obtained from this approach are expected to be applicable to an expanded range of applications.

For the remainder of this paper, Sec. 2 first summarizes the fundamentals of velocity measurements based on TDLAS, with an emphasis on introducing two approaches of obtaining the Doppler shift: the scanned direct-absorption (DA) approach and the wavelength-modulation $(2 f)$ approach. Section 3 analyzes the effects caused by nonuniformities typically encountered in scramjet flows. Results are reported to illustrate the measurement uncertainties caused by boundary effects, divergence/convergence of the measurement region, and the variation flow properties. Section 4 applies the results obtained in Sec. 3 to experimental data to illustrate the usefulness of the results in interpreting practical measurement data.

\section{Principle of TDLAS Velocimetry}

The use of TDLAS was first demonstrated by [11] and thorough description of the technique can be found in [12]. A brief summary here is provided to facilitate the discussion in subsequent sections. Figure 1 illustrates a typical setup of TDLAS-based

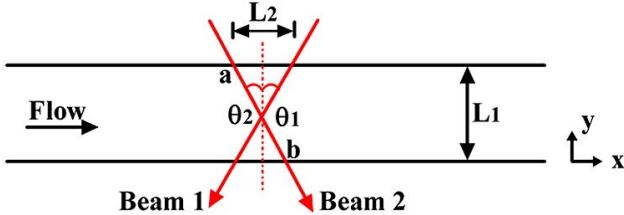

Fig. 1. (Color online) Schematic of TDLAS velocimetry.

velocimetry. A laser beam was split to two probing beams, labeled as beam 1 and beam 2, respectively, in Fig. 1. These probing beams cross in the region of interest and define a plane of measurement. Two angles, $\theta_{1}$ and $\theta_{2}$, are then defined in the plane of measurement to specify the direction of the probing beams relative to the flow (assumed to have uniform velocity and pressure distributions). This work defines $\theta_{1}$ and $\theta_{2}$ as the angles between the laser beams and the direction normal to the flow. To facilitate the discussion, the direction of the flow is taken to be the $x$ direction, and direction normal to the flow the $y$ direction.

The wavelength of the laser is modulated to scan an absorption feature of a target species, typically water vapor in combustion flows. The absorption feature measured by the two probing beams, due to their different orientation relative to the direction of the overall flow, will show a frequency shift because of the Doppler effects. The frequency shift is proportional to the velocity of the flow $(V)$ as shown by the following equation:

$$
\Delta \nu_{\text {Doppler }}=\frac{V}{c} \tilde{\nu}_{0}\left(\sin \theta_{1}+\sin \theta_{2}\right),
$$

where $\Delta \nu_{\text {Doppler }}$ is the Doppler shift between the absorption features measured by the two probing beams, $c$ the speed of light, and $\tilde{\nu}_{0}$ is the line-center frequency of the absorption line. Practical implementation often sets $\theta_{1}=\theta_{2}$, and then Eq. ( 1 ) becomes

$$
\Delta \nu_{\text {Doppler }}=\frac{2 V}{c} \tilde{\nu}_{0} \sin \frac{\theta}{2},
$$

where $\theta=\theta_{1}+\theta_{2}$.

In the presence of nonuniformity along the path of the probing beams, Eqs. (1) and (2) need to be modified and the modification is detailed in Appendix A. The nonuniformity could be in terms of temperature, pressure, and/or velocity. Furthermore, such nonuniformity also causes distortion in the shape of absorption feature, leading to uncertainties in determining the absorption peak and eventually in determining the Doppler shift. Analyzing such uncertainties in TDLAS-based velocimetry is the focus of this work.

In our analyses, we decoupled the nonuniformities to provide insights of the uncertainties and to facilitate the application of the results in practice. The nonuniformities are decoupled into those along the direction of the flow (i.e., the $x$ direction shown in Fig. 1), and those normal to the flow (i.e., the $y$ direction). In practice, nonuniformities in the $y$ direction 
are typically caused by the existence of boundary layers, and those in the $x$ direction are caused by the divergence/convergence of the flow, variations of the boundary layer thickness along the $x$ direction, and/or variations in flow properties due to heat transfer. In this work, uncertainty due to each type of nonuniformity is analyzed in isolation, based on which it is straightforward to analyze multiple types of nonuniformity simultaneously, as illustrated in Sec. 4 .

Before proceeding further, the assumptions invoked in this work are summarized. This work assumes a rectangular flow cross-section and the plane of the optical beams is immune from corner flow. Furthermore, the flow is assumed to be unseparated.

As shown in Eqs. (1) and (2), TDLAS-based velocimetry essentially measures the Doppler shift $\left(\Delta \nu_{\text {Doppler }}\right)$ from the absorption signals registered at the two probing beams. And several approaches exist in practice to obtain the Doppler shift. This work analyzes two of them: DA and $2 f$. In the DA approach, the absorption spectra at both probing beams are first calculated, and then $\Delta \nu_{\text {Doppler }}$ is determined by the frequency difference of an absorption peak on those two spectra. The DA approach is simple when the absorption spectra can be obtained, which requires that a baseline can be obtained [13]. In contrast, the $2 f$ approach utilizes the peaks in the second harmonic of the signals at both probing beams to determine $\Delta \nu_{\text {Doppler }}$ [14]. Compared with the DA approach, the $2 f$ approach can significantly improve the signal-to-noise ratio (SNR) and is therefore suitable for applications with weak absorption, and does not require a baseline under optimized modulation parameters, and is therefore applicable under high pressure $[\underline{15}, \underline{16}]$. A key parameter in the $2 f$ approach is the so-called modulation depth, and this work uses a modulation-depth of 2.2 following [17]. Detailed descriptions of simulating the absorption features in nonuniform flow field and obtaining the peaks are provided in Appendix A for both the DA and $2 f$ methods.

\section{Uncertainty in Velocity Measurements Caused by Nonuniform Flow Parameters}

As mentioned above, this work decouples the nonuniformities in the $x$ direction from those in the $y$ direction, which are treated in Sec. 3.A and 3.B, respectively. The analyses were conducted under the context of applications in our scramjet facility as reported in [18], where the typical flow conditions at the measurement location are Mach number of 1.83 , static temperature of $600 \mathrm{~K}$, and static pressure of $1.0 \mathrm{~atm}$. Water vapor absorption feature centered at $7185.597 \mathrm{~cm}^{-1}$ (i.e., $\widetilde{\nu}_{0}$ ) was used in this analysis. This transition has been applied extensively in practice due to its relatively strong absorption strength at high temperatures and its isolation from interfering transitions in its vicinity $[14,18]$.

Relevant spectroscopic parameters were extracted from HITRAN 2008 [19]. The lower state energy of this absorption line is $E^{\prime \prime}=1045 \mathrm{~cm}^{-1}$. To study the impact of $E^{\prime \prime}$ on the TDLAS measurement, we assumed two other values of $E^{\prime \prime}\left(2000\right.$ and $500 \mathrm{~cm}^{-1}$ ) for this same transition while keeeping its linestrength and $\widetilde{\nu}_{0}$ fixed. This is a hypothetical study (in practice, each transition has its own set of $E^{\prime \prime}$, linestrength, and $\widetilde{\nu}_{0}$ ). Nonetheless, the results are valid to illustrate the effects of $E^{\prime \prime}$ because the TDLAS measurements [see Eq. (1)] do not depend on the absorption strength and depend linearly on $\widetilde{\nu}_{0}$ (if the SNR of the measurements is high enough as discussed in Sec. 3.C). Therefore, it is straightforward to extend the results obtained in such hypothetical study to practical applications.

\section{A. Nonuniformity Along y Direction}

Nonuniformity always exists in the $y$ direction due to the boundary layer effects as illustrated in Fig. 2(a). Figure 2(a) shows a top view of a TDLAS-velocimetry applied to a flow with boundary layers. Here the top view defined as the view along a direction normal to the measurement plane. Both velocity and temperature are nonuniform in the boundary layers. Practical applications may have nonunity Prandtl number and different thickness of velocity and temperature boundary layers. It is straightforward to extend the analysis to such cases. To simplify the problem and elucidate the analysis, the velocity and thermal boundary layer are assumed to have the same thickness $(\delta)$ at both the walls. The velocity was assumed to follow a linear distribution in the boundary layer as shown in Fig. 2(b), and to be constant (at $V_{C}=$ $900 \mathrm{~m} / \mathrm{s}$ ) in the core flow. The temperature distribution in the boundary layers was calculated under the isentropic assumption from the velocity distribution

(a)

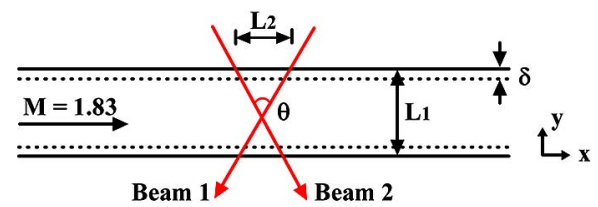

TDLAS-velocity measurement (boundary layer, top view)

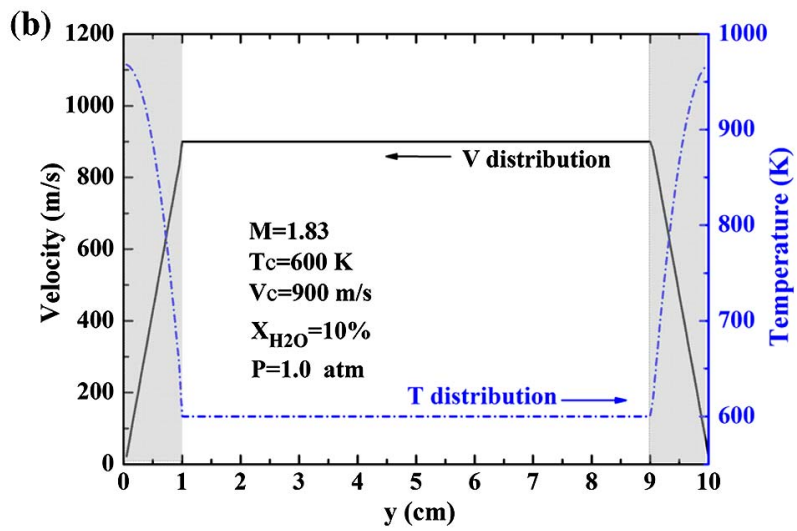

Fig. 2. (Color online) (a) Illustration of TDLAS velocity measurement with boundary layer. (b) Temperature and velocity distributions assumed in the analysis. 
as shown in Fig. 2(b), and the temperature was taken to be constant $\left(T_{C}=600 \mathrm{~K}\right)$ in the core flow. Such an isotropic approximation will exaggerate the effects of the thermal boundary layer, and therefore the uncertainty calculated represents a conservative estimate. The static pressure was assumed to be uniform at $1 \mathrm{~atm}(P=1.0 \mathrm{~atm})$. These conditions correspond to a flow with Mach number (Ma) of 1.83 and a total temperature of $960 \mathrm{~K}$, a representative condition at the inlet of our scramjet combustor [18]. The mole fraction of water vapor was assumed to be uniform at $10 \%(X=10 \%)$ due to the preheating of the inflow by the combustion of hydrogen. These conditions represent a typical flow condition corresponding to the "starting point" of scramjet engine in fly tests [20].

Figure 3 shows the uncertainty in velocity measurements due to the nonuniformity in the boundary layers. Here the uncertainty is defined as

$$
e=\frac{V_{M}-V_{C}}{V_{C}}
$$

where $V_{M}$ is the velocity that the TDLAS sensor would measure. Two approaches, the DA and $2 f$ approaches, of obtaining the Doppler shift were compared for transitions with various lower state energies $\left(E^{\prime \prime}\right)$. Note that the error was negative for all these cases (i.e., $V_{M}$ is less than $V_{C}$ ); the flow is slower in the boundary layer than in the core flow. The results shown in Fig. $\underline{3}$ provide several useful observations.

First, obviously, the error caused by boundary layer increases with the thickness of the boundary relative to width of the core flow (quantified by $\delta / L_{1}$ in Fig. 3). When $\delta / L_{1}=5 \%$ the error can reach $\sim 10 \%$ depending on the specifics of the TDLAS sensor (e.g., absorption transition used and the method used to determined the Doppler shift). Figure $\underline{3}$ shows typical values of $\delta / L_{1}$ in the inlet, combustor, and exit sections of our scramjet facility. These results suggest that the analysis of boundary effects is not trivial, and the simulation results shown here can be used as guidelines.

Second, the errors are smaller when the $2 f$ method was used than when the DA method was used to determine the Doppler shift. Our explanation for the superiority of the $2 f$ method here is that the

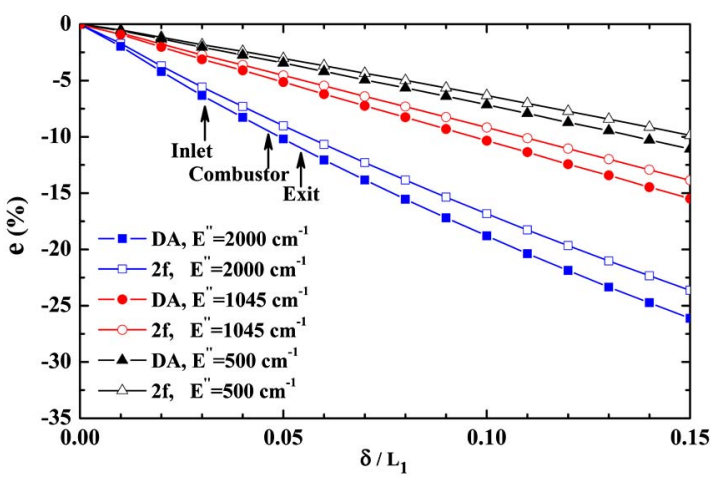

Fig. 3. (Color online) Velocity error caused by nonuniformity in the boundary layers.
$2 f$ method is less sensitive to the distortion in the shape of the absorption feature. As mentioned in Sec. 2 , the nonuniformities in the boundary layers cause distortion in the shape of the absorption feature. As a result, the absorption shape can no longer be perfectly fitted with the Voigt profile. The $2 f$ signal $\left(R_{2 f}\right)$ is mainly determined by the second harmonic of signal $\left(H_{2}\right)$, as shown in Eq. (A13) in Appendix A. It corresponds to the derivative of the absorption shape and is less sensitive to the distortion of the absorption shape.

In the past, the $2 f$ approach has been recognized for being able to improve the SNR compared to the DA approach for TDLAS-based velocimetry [11]. These results suggest that the use of the $2 f$ approach has an additional advantage in TDLAS-based velocimetry: the insensitivity to the interference due to the boundary layers. One factor contributing to such insensitivity is the fact that the $2 f$ method detects the change in the absorption spectra, not the absorption spectra itself [11]. As a result, the $2 f$ method is less sensitive to the distortion in the lineshape caused by boundary layer effects. The results shown in Fig. 4 elucidate such insensitivity. Here, Fig. 4(a) shows the simulated DA and $2 f$ signals at one probe beam according to the method described in Appendix A for a flow with $\delta / L_{1}=15 \%$ and $E^{\prime \prime}=2000 \mathrm{~cm}^{-1}$. The properties of the main flow are those specified in Fig. 2. The DA signal was fit with a Voigt lineshape, which would result in a precise fit should the flow be uniform and have no boundary layer. However, the boundary layer distorts the lineshape, causing a discrepancy between the simulated (or distorted) lineshape and the best Voigt fit. The discrepancy (i.e., the fitting residual) is shown in Fig. 4(b). Similarly, the $2 f$ signal was simulated and fit with a lineshape assuming no boundary layer effects, and the discrepancy between the simulated signal and the best fit are also shown in Fig. $\underline{4(b)}$. The results in Fig. $\underline{4}$

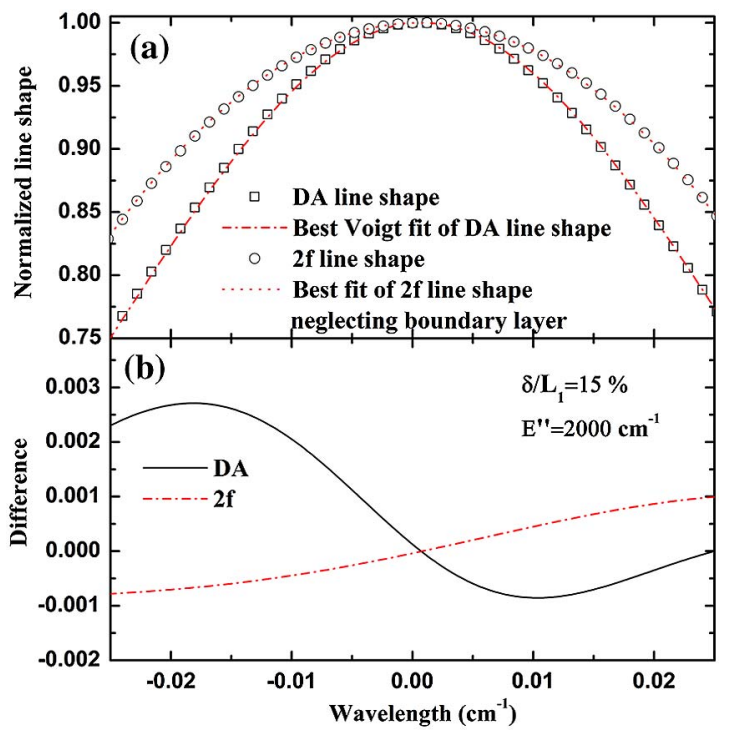

Fig. 4. (Color online) Comparison of the lineshape distortion caused by boundary layer for the DA and $2 f$ methods. 
illustrate that the $2 f$ method is less sensitive to lineshape distortion caused by the boundary layer in two aspects: (1) as seen from Fig. 4(b), the discrepancy for the DA method ranges from $-0.1 \%$ to $+0.3 \%$ of the peak value, while that of the $2 f$ method is within $\pm 0.1 \%$, and (2) the peak locations determined by fitting the DA and $2 f$ signals were, respectively, $3.6 \times 10^{-3} \mathrm{~cm}^{-1}$ and $3.2 \times 10^{-3} \mathrm{~cm}^{-1}$ off the true peak location (i.e., the peak location assuming no boundary layer effects). Calculation at the other probe beam a yielded similar trend; i.e., the $2 f$ signals suffered less lineshape distortion and smaller error in determining the peak location. After calculating the errors in the peak location at both probe beams, the error in velocity shown in Fig. $\underline{3}$ can then be obtained (note that under the conditions used here, the Doppler shift at $900 \mathrm{~m} / \mathrm{s}$ is $0.011 \mathrm{~cm}^{-1}$ ). Calculations were also made at other flow condition (different boundary layer thickness and lower state energies), and the results all show that the $2 f$ method is less sensitive to the lineshape distortion caused by flow nonuniformities, and yields more accurate determination of the peak location than the DA method.

Also note that in practice, the error caused by lineshape distortion as illustrated in Fig. 4 only represents one of the sources of uncertainty. As aforementioned, this work attempts to examine these sources independently first. Section $\underline{4}$ describes example application of the analysis to experimental results where errors caused by multiple sources are considered simultaneously.

Third, the error decreases when transition with smaller $E^{\prime \prime}$ is used if the boundary layer is hotter than the core flow. This is a typical situation for supersonic test facilities, where the boundary layer has higher temperature and lower velocity than the core flow because the temperature drop at the wall due to heat loss is less significant than the temperature rise due to conversion of the kinetic energy. In this case, transitions with smaller $E^{\prime \prime}$ are advantageous, because it increases the absorption due to the cooler core flow, and decreases the absorption due to the hotter boundary layers, resulting in improved accuracy of velocity measurements. In applications where the boundary layer is cooler than the core flow, the effects of $E^{\prime \prime}$ are reversed, and an elaborated discussion can be found in [2].

\section{B. Nonuniform Along $x$ Direction}

Nonuniformities along the $x$ direction also occur in supersonic flows due to a variety of reasons. A shockwave intercepting the probing beams represents an extreme case of nonuniformity along the $x$ direction. Here, we analyze effects caused by other factors including the divergence/convergence of the flow, variations of the boundary layer thickness, and/or variations in flow properties due to heat release.

First, we consider the effects caused by the convergence/divergence of the flow, as schematically shown in Fig. 5 , where a TDLAS-based velocimetry is applied to measure the velocity in the section with a

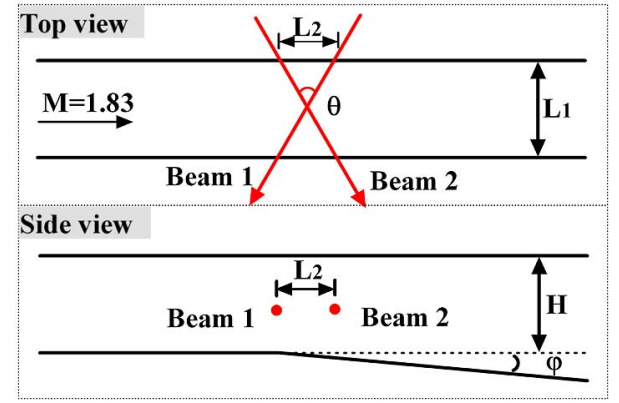

Fig. 5. (Color online) Illustration of TDLAS velocity measurement with flow divergence.

diverging angle of $10 \mathrm{deg}(\varphi=10 \mathrm{deg})$. The analysis of a converging section is similar and therefore not presented here. In this case, the flow properties vary along the $x$ direction and the variations were modeled by assuming an isentropic flow. Figure 6 shows the distribution of velocity $(V)$, temperature $\overline{(T)}$, and pressure $(P)$ using the incoming flow conditions as those shown in Fig. 2(b). The height of the crosssection, labeled as $H$ in Fig. 5 , was taken to be $5 \mathrm{~cm}$. Figure 7 shows the error in velocity measurements caused by such nonuniformities. Here, the error is defined as,

$$
e=\frac{V_{M}-V_{\mathrm{avg}}}{V_{\mathrm{avg}}},
$$

where $V_{\text {avg }}$ is the arithmetically averaged velocity along $L_{2}$. Note that Eqs. (3) and (4) are independent: they considers the uncertainty caused by nonuniformities in the $x$ and $y$ directions, respectively. The combined velocity error caused by nonuniformities in both directions is considered in Sec. $\underline{4}$.

As shown in Fig. 7, velocity error is generally within $\pm 1 \%$ when the divergence angle is less than 20 deg. For practical scramjet applications, the divergence angle in the combustor and exit is normally less than $5 \mathrm{deg}$ as indicated in Fig. 7 , which results in a velocity error less than $0.2 \%$ for the conditions shown here. Two interesting observations can be made from these results. First, such an error is

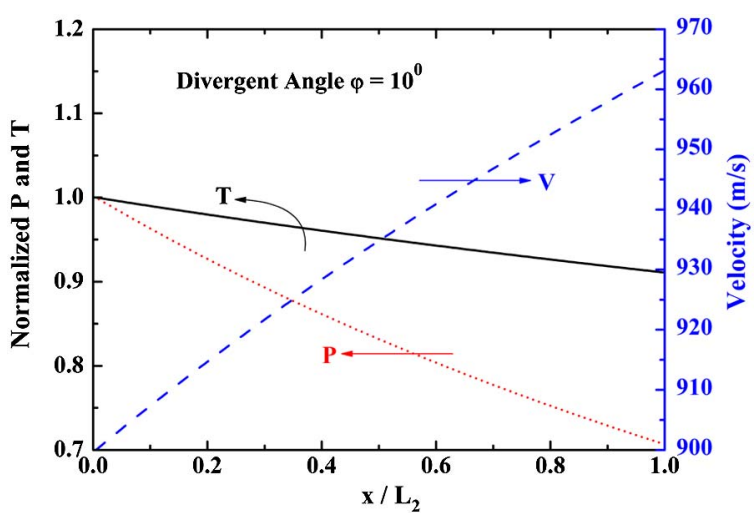

Fig. 6. (Color online) Temperature, pressure, and velocity distribution assumed in the analysis. 


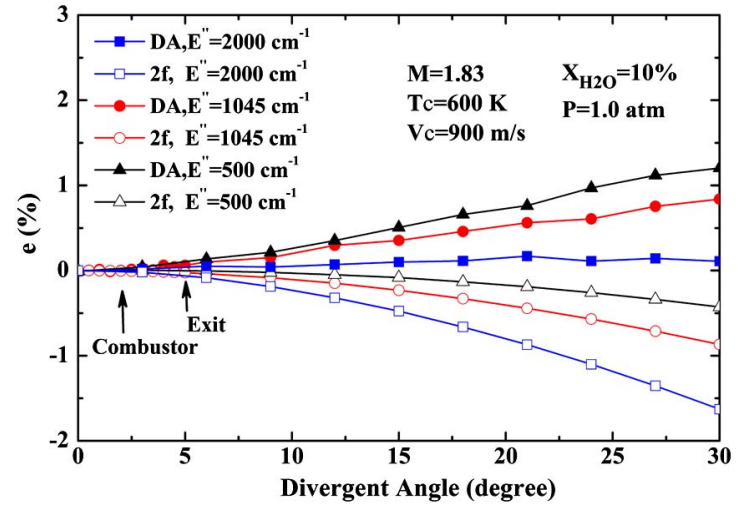

Fig. 7. (Color online) Velocity error caused by flow divergence.

significantly smaller than that caused by nonuniformity in the $y$ direction as discussed in Sec. 3.A for typical scramjet flows, and second, such an error is also significantly smaller than the variation of $V$ itself in the $x$ direction (as shown in Fig. $\underline{7}, V$ itself varies for about $7 \%$ along $L_{2}$ ). Our explanation for these two observations is that the probing beams cross the flow at an angle and measure a velocity that is a weighted averaged of absorption coefficient $(k)$ along $L_{2}$. Even though this average is not an arithmetic average, it is very close to the arithmetic average. The following results will show that both observations remain valid for more general cases where nonuniformities exist in multiple parameters along the $x$ direction.

In practice, other factors can cause nonuniformities along the $x$ direction in addition to the divergence/ convergence of the flow. For example, in scramjet combustors, the static temperature and velocity along the $x$ direction may vary because of heat release or the increasing thickness of the boundary layers. In this work, we analyzed a simple case where the static temperature $(T)$, pressure $(P)$, and velocity $(V)$ all vary along the $x$ direction in a linear fashion, as shown in Fig. 8. The rate of variation for the static temperature, pressure, and velocity are $k_{T}, k_{P}$, and $k_{V}$, respectively. Such a linear variation represents a good approximation for the conditions in our scramjet facility, and the

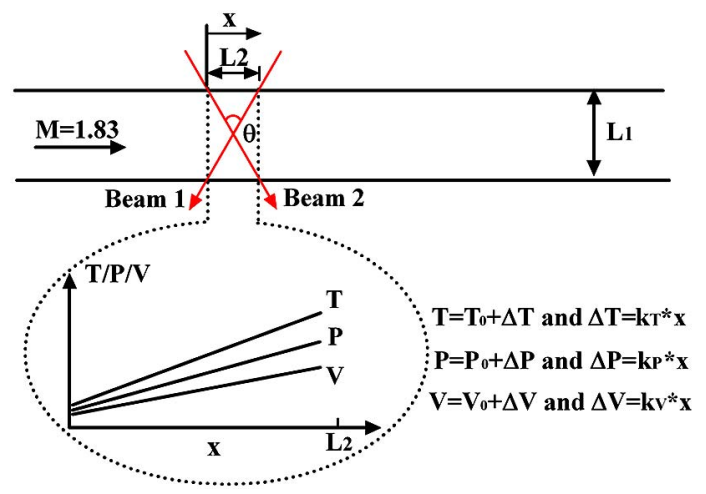

Fig. 8. (Color online) Illustration of TDLAS velocity measurement with nonuniformity simultaneously present in temperature, pressure, and velocity along $L_{2}$. variations (represented by $\Delta T, \Delta P$, and $\Delta V$ in Fig. 8) along $L_{2}$ typically do not exceed $20 \%$ of the incoming conditions at $x=0$ (represented by $T_{0}, P_{0}$, and $V_{0}$ in Fig. 8).

Figure 9 shows the error caused by such simultaneous variation in $T, P$, and $V$ along the $x$ direction. The definition of the error is the same as in Eq. (4), defined as the difference between the measured velocity relative to the arithmetic average velocity along $L_{2}$ as the reference. Figure 9(a) shows the error when the DA approach was used, and Fig. 9(b) when the $2 f$ method was used. In Fig. $\underline{9}, \Delta V$ was taken to be $0.2 \times V_{0}$, corresponding to the scenario when the maximum amount of nonuniformity in $V$ was reached. Again, we see that the error is significantly smaller than both the error caused by nonuniformities in the $y$ direction and the variation of the velocity along $L_{2}$ (20\% in this case).

Similar plots can be generated to show the error versus the variation of $V$. To aid the visualization of the results, Fig. 10 shows the maximum absolute error (i.e., $\max |e|)$ when $T, P$, and $V$ all vary within $\pm 20 \%$ of their incoming values. As shown, the maximum error of velocity is less than $1.2 \%$, indicating that even the flow parameter varies substantially along $L_{2}$ (normally smaller than $5 \mathrm{~cm}$ ); the velocity error remains less than $1 \%$ relative to the arithmetic average if a suitable absorption line is used.

\section{Consideration of SNR}

Analysis thus far has been made under the assumption of infinite SNR. In practice, the measurements have finite SNR due to measurement noises from various sources, such as laser intensity fluctuation,
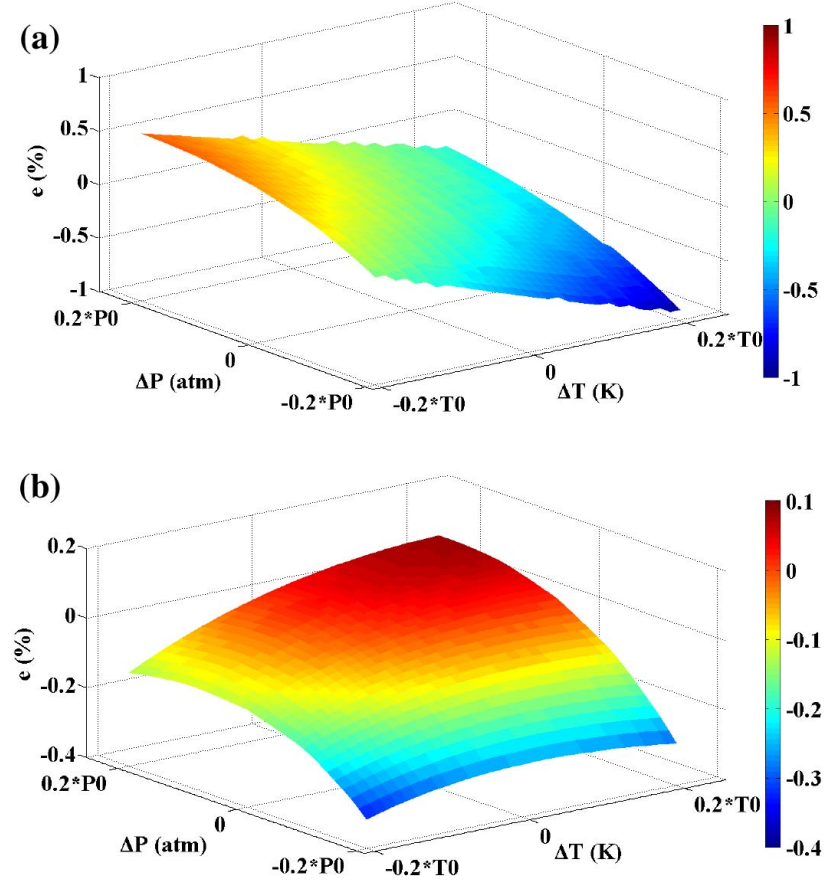

Fig. 9. (Color online) (a) Velocity error versus due to variation of $T$ and $P$ when the DA method is used. (b) Velocity error versus due to variation of $T$ and $P$ when the $2 f$ method is used. 


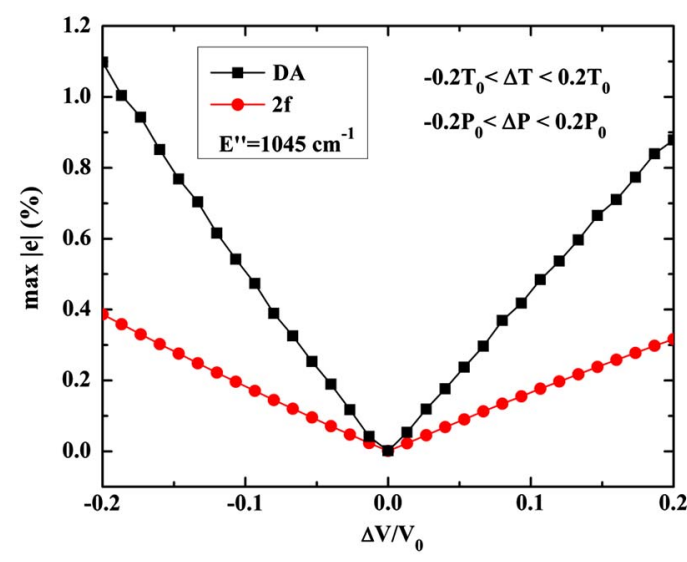

Fig. 10. (Color online) Maximum velocity measurement error versus $k_{V}$.

detector noise, and digitization noise, etc., and the measurement noises will cause uncertainty in velocity measurements in addition to those caused by flow nonuniformities. A full-length discussion of the effect of SNR merits a separate paper. Here we briefly explain our method of considering SNR and report some sample results.

Our analysis considered the combined effects of all noise in the transmitted laser intensities (which is the signal used to infer velocity) according to the following equation:

$$
I_{t}^{m}(\nu)=I_{t}(\nu)+I_{n}(\nu),
$$

where $I_{t}^{m}$ is the transmitted laser intensity measured by the detector. The measurements are composed of two parts: the "real" transmitted laser intensity $\left(I_{t}\right)$ and measurement noises due to various sources $\left(I_{n}\right)$. Then the SNR of the measurement is defined as

$$
\mathrm{SNR}=I_{t} / I_{n} .
$$

In the analysis presented in this paper, we assumed the measurement noise to be random, which follows a normal distribution. The SNR of the measurements is then determined by the magnitude of the normal distribution. Under this assumption, the analysis can be performed by generating random noises $\left(I_{n}\right)$ artificially and adding them to the signal $\left(I_{t}\right)$ to obtain the simulated measurements $\left(I_{t}^{m}\right)$. The simulated measurements can then be analyzed using the procedure detailed in Appendix A to evaluate the effects of SNR in combination of flow nonuniformities. Elaborated models can also be developed to simulate realistic noises that do not follow a normal distribution. As mentioned before, such full-length discussion merits a separate treatment. Here, we analyze an ideal case to illustrate the effects of measurement SNR.

The case analyzed here involves a uniform flow (i.e., without any boundary layer effects), and the flow conditions are the main flow conditions shown in Fig. 2. The transitions used are the same ones

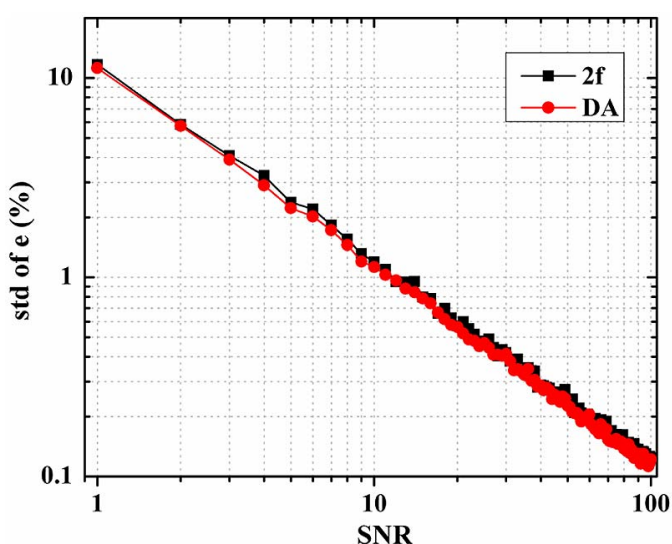

Fig. 11. (Color online) Relationship between velocity error and signal-to-noise ratio (SNR).

used in previous sections with $E^{\prime \prime}=1045 \mathrm{~cm}^{-1}$. The results are shown in Fig. 11. Due to the statistical nature of the noise, in Fig. 11, 300 simulations were performed at each SNR; the relative error $(e)$ is calculated in each simulation, and the standard deviation (std) of all 300 simulations is plotted in Fig. 11. We conducted analysis under other flow conditions, and the results shown in Fig. 11 are quite insensitive to the mean flow properties.

Several observations can be made from Fig. 11. First, it shows that the error decreases as SNR increases as intuitively expected. Second, it shows that for a SNR larger than 10, the error caused by measurement noise is $\sim 1 \%$ for both the DA and $2 f$ methods. For the experimental data presented in Sec. 4 , the SNR of our measurements are in the range from 20 to 30 . Therefore, we expect the uncertainty to be dominated by flow nonuniformities under these experimental conditions. Third, for velocity measurements, Fig. 11 does not suggest a dramatic improvement from the $2 f$ method in accuracy relative to the DA method in the presence of measurement noise.

\section{Application of Analysis to Experimental Data}

This section applies the analysis presented above to experimental data obtained in our scramjet facility $[18,21]$. The scramjet facility is a direct-connected facility capable of simulating supersonic combustion under flight condition. Experiments were performed in a two-dimensional combustor fueled with ethylene, with a test section of $40 \times 85 \mathrm{~mm}^{2}$. The detailed description of this facility and the experiments can be found in [21].

Five representative cases were examined and the results are summarized in Figs. 12 and 13 . These five cases include velocity measured at the inlet (case 1) and the exit (case 2) of the scramjet combustor at Mach 1.8, and velocity measured at the inlet (case 3), the combustor (case 4), and the exit (case 5) of the scramjet at Mach 2.5. Figure 12 shows the relative error in velocity for each case; the top panel of Fig. 13 shows the velocity and the bottom panel the absolute error. 


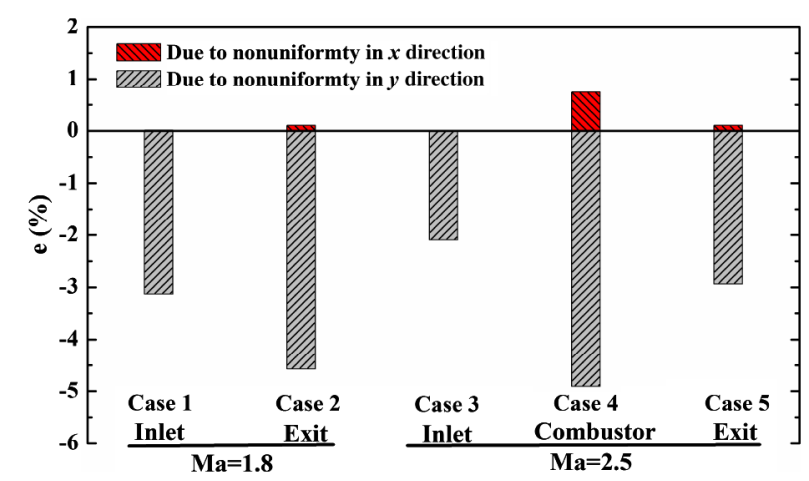

Fig. 12. (Color online) Velocity uncertainty of experimental results caused by nonuniformity along $L_{1}$ and $L_{2}$.

These error analyses were performed using the results shown in Figs. 3, 7, and 10. Here we use case 4 as an example to elucidate the steps of our analyses. First, the thickness of the boundary layer $(\delta)$ is estimated to be $4 \mathrm{~mm}$ for the combustor of our direct-connected scramjet facility according to CFD simulations [22]. The measurement pathlength $\left(L_{1}\right)$ was $85 \mathrm{~mm}$. Therefore from Fig. 3, the error caused by the boundary layer in this case is estimated to be $4.8 \%$ (the DA method was used with $E^{\prime \prime}=$ $1045 \mathrm{~cm}^{-1}$ in this measurement). Second, the divergence angle in the experiments was $2 \mathrm{deg}$. Then from Fig. 7, the error caused by the divergence angle in this case was estimated to be $0.1 \%$. Third, along the $L_{2}$ direction, the variation of the parameters in the combustor was estimated to be $\Delta T<0.1 \times T_{0}$, $\Delta P<0.2 \times P_{0}, \quad \Delta V<0.05 \times V_{0}$. Therefore, using the results shown in Figs. 7 and 10 , velocity error caused by nonuniformities along the $L_{2}$ direction was determined to be $1 \%$.

With the above relative errors and the velocity shown in the top panel of Fig. 12 , it is straightforward to estimate the absolute error shown in the bottom panel of Fig. 12. For example, for case 4, the measured velocity (nominal velocity) was $560 \mathrm{~m} / \mathrm{s}$, and the absolute errors were estimated to be

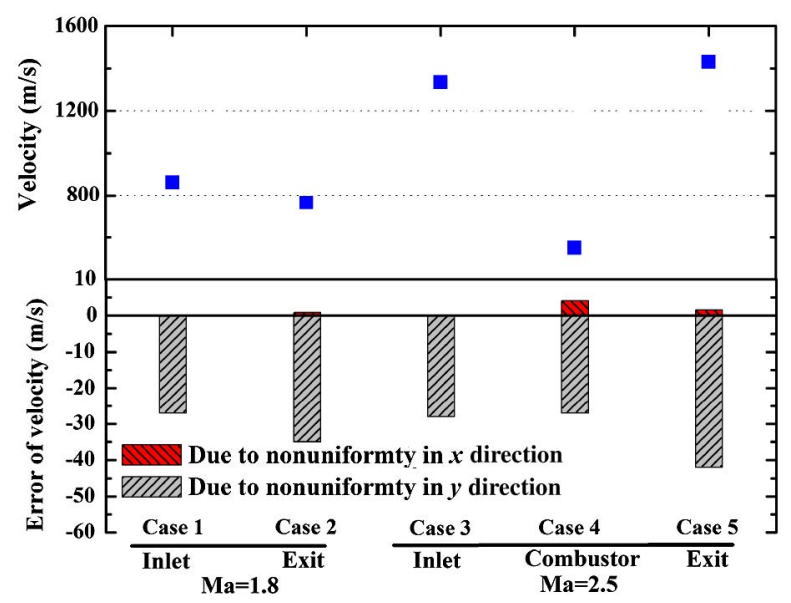

Fig. 13. (Color online) Velocity uncertainty analysis for experimental results in scramjet combustor. $\sim 22 \mathrm{~m} / \mathrm{s}$, in which $-27 \mathrm{~m} / \mathrm{s}$ was caused by the nonuniformities along the $y$ direction (i.e., boundary layer effects), and $5 \mathrm{~m} / \mathrm{s}$ was caused by the nonuniformities along the $x$ direction. The error analyses for other cases were estimated similarly.

From these results, it can be concluded that that the error in scramjet flows is mainly due to the nonuniformities in the boundary layers. Moreover, the flow fields were assumed to be quasi-twodimensional in this work; i.e., the flow parameters along $L_{1}$ are uniform except in the boundary layers. When significant three-dimensional effects exist (e.g., when a shock wave intercepts the probe beams), both the overall error and the relative contribution due to $x$ - and $y$-direction nonuniformities will be different than those reported here, and the interpretation of the TDLAS measurements is more complicated [10]. Lastly, the assumptions made in our analysis should be iterated, including the neglect of corner-flow effects and unseparated flow condition. Also, this work focused on the impact of flow nonuniformities and neglected other types of uncertainties. For example, in practice, beamsteering and the uncertainty in the angle $(\theta)$ between beams (e.g., caused by mechanical vibration and thermal expansion) can lead to additional errors. Our method essentially divides errors into two categories: those caused by flow nonuniformities and those caused by other effects. The later categories were treated as measurement noises and discussed in Sec. 3.C.

\section{Summary}

This work investigated the uncertainty in velocity measurements using TDLAS-based techniques under the context of scramjet flows. This study focuses on quantifying the uncertainties caused by nonuniformities in the flow. The nonuniformities typically encountered in practice were decoupled into those in the direction normal to the mean flow (i.e., boundary layer effects the $y$ direction) and those in the direction along the mean flow (the $x$ direction). Two variations of TDLAS, the DA and $2 f$, were examined.

Two main findings can be summarized from the results obtained in this study. First, under typical conditions in scramjet flows, the error caused by the nonuniformities in $x$ direction is significantly smaller than that caused by the boundary layer effects and also than the variation of the velocity itself in the $x$ direction. Second, the $2 f$ method is less sensitive to the boundary layer effects than the DA method in determining the Doppler shift. The boundary layers effects distort the absorption line shape to deviate from the Voigt profile. Unlike the DA method, the $2 f$ method measures the second harmonic of the absorption feature. It detected the peak by analyzing the derivative of the absorption shape and is less sensitive to the distortion of the absorption shape. As a result, in our results, the $2 f$ method yielded more accurate Doppler shift than the DA method. 
These findings were then applied to analyze the experimental data obtained in a scramjet facility, illustrating the usefulness of the results reported. We expect this work to be valuable for quantifying the experimental data, the data analysis, and also the design and optimization of TDLAS-based velocimetry for high speed flows.

\section{Appendix A: Description of the DA and $2 f$ methods}

In a nonuniform flow, the absorption of laser radiation is described by the Beer-Lambert as shown below:

$$
\tau(\nu)=\frac{I(\nu)}{I_{0}(\nu)}=\exp \left(-\int_{a}^{b} k(\nu, l) \mathrm{d} l\right),
$$

where $\tau(\nu)$ is the fractional transmission at frequency $v, I$ and $I_{0}$ are the transmitted and incident laser intensities, respectively, $k$ is the spectral absorption coefficient, $l$ defines a one-dimensional coordinate along the path length, and $a$ and $b$ are the beginning and the end of the light path as shown in Fig. 1 . Absorption coefficient $k$ is calculated as

$$
k(\nu, l)=S[T(l)] \cdot P(l) \cdot X(l) \cdot \phi\left[\nu-\nu_{0}(l)\right],
$$

where $S[T(l)]$ is the line-strength and $T(l)$ emphasizes the nonuniformity of the flow (i.e., $T$ depends on $l$, and the same notation is used hereafter), $P(l)$ the static pressure, $X(l)$ the mole fraction of the absorbing species (i.e., water vapor in this present paper), and $\phi$ is the line-shape function. As discussed in Sec. 2, is the frequency of the line center in the presence of Doppler and pressure-induced shift:

$$
\nu_{0}(l)=\begin{array}{ll}
\tilde{\nu}_{0}+\tilde{\nu}_{0} \cdot \frac{V(l)}{c} \cdot \sin \frac{\theta}{2}+\delta \cdot P(l) & \text { for Beam1 } \\
\tilde{\nu}_{0}-\tilde{\nu}_{0} \cdot \frac{V(l)}{c} \cdot \sin \frac{\theta}{2}+\delta \cdot P(l) & \text { for Beam2 }
\end{array},
$$

where $\delta$ the coefficient of pressure-induced shift and other notations have been introduced in Sec. 1 already.

Combining Eqs. (A1) and (A2), the fractional transmission becomes

$$
\tau(\nu)=\exp \left(-\int_{a}^{b} S[T(l)] \cdot P(l) \cdot X(l) \cdot \phi\left[\nu-\nu_{0}(l)\right] \mathrm{d} l\right) .
$$

Note that in Eq. (A4), the only term in the integral that depends on $\nu$ is the lineshape function. Therefore, Eq. (A4) can be rewritten as

$$
\tau(\nu)=\exp (-A \cdot \bar{\phi}(\nu)),
$$

where $A$ absorbs the terms that have no $\nu$-dependence $(S, P$, and $X)$ and is essentially the magnitude of the lineshape as shown below:

$$
A=\int_{-\infty}^{+\infty} \tau(\nu) \mathrm{d} \nu
$$

Following Eq. (A5), $\bar{\phi}(\nu)$ is a normalized lineshape such that $\int_{-\infty}^{+\infty} \overline{\phi(\nu)} \mathrm{d} \nu=1$.

Based on the above understanding, this work calculated Eq. (A4) numerically along the line-of-sight (i.e., $l$ ) based on the given distribution of flow parameters (i.e., temperature, pressure, and velocity). Once Eq. (A4) is evaluated, $\bar{\phi}(\nu)$ can be obtained following Eqs. (A4) and (A5).

Once $\bar{\phi}(\nu)$ is obtained, the DA method seeks the peak of $\bar{\phi}(\nu)$ In this analysis, the peak location was determined by first coarsely locating the region where the peak resides, then fitting this region using a second-order polynomial to obtain an accurate peak location. Finally, the peak frequency of $\bar{\phi}(\nu)$ of beams 1 and 2 is then used to calculate the frequency shift and velocity according to Eq. (2).

In contrast, the $2 f$ method utilizes the peaks in the second harmonic of the transmitted signal to determine frequency shift. In practice, the $2 f$ method modulates the laser with a sinusoidal wave on top of a triangle wave. The output frequency of the laser can be expressed by

$$
\nu(t)=\bar{\nu}+a \cos \left(2 \pi f_{m} t\right),
$$

where $\bar{\nu}$ is the center laser frequency, $a$ is the modulation amplitude, and $f_{m}$ is the frequency of the sinusoidal modulation. Note Eq. (A7) neglects the effects due to the triangle modulation since the $2 f$ signal is detected only at the frequency of the sinusoidal modulation $\left(f_{m}\right)$, which is typically significantly higher than that of the triangle modulation.

To analyze the $2 f$ signal, Eq. (A7) is used to transform Eq. (A4) from the frequency domain into the time domain so that a Fourier expansion can be performed as shown below:

$$
\begin{aligned}
\tau(\bar{\nu}(t)) & =\tau\left(\bar{\nu}+a \cos \left(2 \pi f_{m} t\right)\right) \\
& =-\sum_{n=0}^{n=+\infty} H_{n}(\bar{\nu}, a) \cos \left(n 2 \pi f_{m} t\right),
\end{aligned}
$$

where the $H_{s}$ are the Fourier coefficients, and they are given by

$$
H_{0}(\bar{\nu}, a)=-\frac{1}{2 \pi} \int_{-\pi}^{+\pi} \tau(\bar{\nu}+a \cos \theta) \cdot \mathrm{d} \theta,
$$

$$
H_{n}(\bar{\nu}, a)=-\frac{1}{\pi} \int_{-\pi}^{+\pi} \tau(\bar{\nu}+a \cos \theta) \cdot \cos n \theta \cdot \mathrm{d} \theta(n \geq 1),
$$

The final $2 f$ signal $\left(R_{2 f}\right)$ is then [15]

$$
R_{2 f}=\left(X_{2 f}^{2}+Y_{2 f}^{2}\right)^{1 / 2}
$$

while $X_{2 f}$ and $Y_{2 f}$ are given by 


$$
\begin{aligned}
& X_{2 f}=\frac{G \bar{I}_{0}}{2}\left[H_{2}+\frac{i_{0}}{2}\left(H_{1}+H_{3}\right) \cos \omega\right], \\
& Y_{2 f}=-\frac{G \bar{I}_{0}}{2} \cdot \frac{i_{0}}{2}\left(H_{1}-H_{3}\right) \sin \omega,
\end{aligned}
$$

where $G$ is the optical-electrical gain of the detection system and $\omega$ the phase shifts between the laser intensity modulation and wavelength modulation (typically equal to $\pi$ in practice). Note that the above discussion is based on the assumption that the $2 f$ method completely removes the baseline. This assumption is valid when the baseline is constant or a linear function of frequency, which can be realized in many applications by optimizing the modulation frequency and modulation depth.

To summarize, this work analyzed the $2 f$ method in the following steps. First, the fractional transmission was calculated according to Eq. (A4) numerically. Second, the results in step 1 were then transformed into time domain to perform a Fourier expansion according to Eqs. (A8-A10). Third, the first three Fourier coefficients $\left(\overline{H_{1}}, \overline{H_{2}}\right.$, and $\left.H_{3}\right)$ were then used to calculate the $2 \mathrm{f}$ signal $\left(R_{2 f}\right)$ according to Eqs. (A11-A13). Lastly, the peak of $R_{2 f}$ was determined using the same method as in the DA method to calculate the frequency shift and the velocity. We set $G=1 \mathrm{~V} / \mathrm{M}$ (volts per watt) in all analysis. Note that $G$ only scales the magnitude of the $2 f$ signal and does not affect the analysis unless SNR is considered, as briefly discussed in Sec. 3.C. We also set $i_{0}=$ 0.1 and $\omega=\pi$ to simulate typical practical conditions.

\section{References}

1. R. K. Hanson, "Applications of quantitative laser sensors to kinetics, propulsion and practical energy systems," Proc. Combust. Inst. 33, 1-40 (2011).

2. X. Ouyang and P. L. Varghese, "Line of sight absorptionmeasurements of high-temperature gases with thermal and concentration boundary layers," Appl. Opt. 28, 3979-3984 (1989).

3. W. W. Cai, D. J. Ewing, and L. Ma, "Application of simulated annealing for multispectral tomography," Comput. Phys. Commun. 179, 250-255 (2008).

4. S. T. Sanders, J. Wang, J. B. Jeffries, and R. K. Hanson, "Diodelaser absorption sensor for line-of-sight gas temperature distributions," Appl. Opt 40, 4404-4415 (2001).

5. X. Liu, J. B. Jeffries, and R. K. Hanson, "Measurement of nonuniform temperature distributions using line-of-sight absorption spectroscopy," AIAA J. 45, 411-419 (2007).

6. A. D. Griffiths and A. F. P. Houwing, "Diode laser absorption spectroscopy of water vapor in a scramjet combustor," Appl. Opt. 44, 6653-6659 (2005).

7. G. B. Rieker, J. B. Jeffries, R. K. Hanson, T. Mathur, M. R. Gruber, and C. D. Carter, "Diode laser-based detection of combustor instabilities with application to a scramjet engine," Proc. Combust. Inst. 32, 831-838 (2009).

8. L. S. Chang, C. L. Strand, J. B. Jeffries, and R. K. Hanson, "Supersonic mass flux measurements via tunable diode laser absorption and non-uniform flow modeling," in Proceedings of 49th AIAA Aerospace Sciences Meeting including the New Horizons Forum and Aerospace Exposition (American
Institute of Aeronautics and Astronautics, 2011), paper AIAA 2011-1093.

9. L. S. Chang, J. B. Jeffries, and R. K. Hanson, "Mass flux sensing via tunable diode laser absorption of water vapor," AIAA J. 48, 2687-2693 (2010).

10. M. S. Brown and T. F. Barhorst, "Post-flight analysis of the diode-laser-based mass capture experiment onboard HIFiRE flight 1," in Proceedings of 17th AIAA International Space Planes and Hypersonic Systems and Technologies Conference (American Institute of Aeronautics and Astronautics, 2011), paper AIAA-2011-2359.

11. L. C. Philippe and R. K. Hanson, "Laser diode wavelengthmodulation spectroscopy for simultaneous measurement of temperature, pressure, and velocity in shock-heated oxygen flows," Appl. Opt. 32, 6090-6103 (1993).

12. M. P. Arroyo, S. Langlois, and R. K. Hanson, "Diode-laser absorption technique for simultaneous measurements of multiple gasdynamic parameters in high-speed flows containing water vapor," Appl. Opt. 33, 3296-3307 (1994).

13. J. A. Silver and D. J. Kane, "Diode laser measurements of concentration and temperature in microgravity combustion," Meas. Sci. Technol. 10, 845-852 (1999).

14. J. T. C. Liu, G. B. Rieker, J. B. Jeffries, M. R. Gruber, C. D. Carter, T. Mathur, and R. K. Hanson, "Near-infrared diode laser absorption diagnostic for temperature and water vapor in a scramjet combustor," Appl. Opt. 44, 6701-6711 (2005).

15. H. Li, G. B. Rieker, X. Liu, J. B. Jeffries, and R. K. Hanson, "Extension of wavelength-modulation spectroscopy to large modulation depth for diode laser absorption measurements in high-pressure gases," Appl. Opt. 45, 1052-1061 (2006).

16. G. B. Rieker, J. B. Jeffries, and R. K. Hanson, "Calibration-free wavelength-modulation spectroscopy for measurements of gas temperature and concentration in harsh environments," Appl. Opt. 48, 5546-5560 (2009).

17. X. Zhou, J. B. Jeffries, and R. K. Hanson, "Development of a fast temperature sensor for combustion gases using a single tunable diode laser," Appl. Phys. B 81, 711-722 (2005).

18. F. Li, X. Yu, H. Gu, Z. Li, L. Chen, and X. Chang, "Measurement of temperature, velocity, and water vapor concentration in a scramjet combustor based on near-infrared diode laser absorption," in Proceedings of Seventeenth AIAA International Space Planes and Hypersonic Systems and Technologies (American Institute of Aeronautics and Astronautics, 2011), paper AIAA 2011-2214.

19. L. S. Rothman, I. E. Gordon, A. Barbe, D. ChrisBenner, P. F. Bernath, M. Birk, V. Boudon, L. R. Brown, A. Campargue, J.-P. Champion, K. Chance, L. H. Coudert, V. Dana, V. M. Devi, S. Fally, J.-M. Flaud, R. R. Gamache, A. Goldman, D. Jacquemart, I. Kleiner, N. Lacome, W. J. Lafferty, J.-Y. Mandin, S. T. Massie, S. N. Mikhailenko, C. E. Miller, N. Moazzen-Ahmadi, O. V. Naumenko, A. V. Nikitin, J. Orphal, V. I. Perevalov, A. Perrin, A. Predoi-Cross, C. P. Rinsland, M. Rotger, M. Simečková, M. A. H. Smith, K. Sung, S. A. Tashkun, J. Tennyson, R. A. Toth, A. C. Vandaele, and J. VanderAuwera, "The HITRAN 2008 molecular spectroscopic database," J. Quant. Spectrosc. Radiat. Transfer 110, 533-572 (2009).

20. K. N. Roberts and D. R. Wilson, "Analysis and design of a hypersonic scramjet engine with a transition Mach number of 4.00," presented at the47th AIAA Aerospace Sciences Meeting Including The New Horizons Forum and Aerospace Exposition, Orlando, Florida, 5-8 January 2009.

21. F. Li, X. Yu, H. Gu, Z. Li, Y. Zhao, L. Ma, L. Chen, and X. Chang, "Simultaneous measurements of multiple flow parameters for scramjet characterization using tunable diodelaser sensors," Appl. Opt. 50, 6697-6707 (2011).

22. T. Wan, L. Chen, J. Wang, X. Fan, and X. Chang, "Computational study of supersonic combustion using three kerosene reaction mechanism models," presented at the13th Asian Congress of Fluid Mechanics, Dhaka, Bangladesh, 17-21 December 2010. 